\title{
Composição química e atividade antioxidante do óleo volátil de Eupatorium polystachyum DC.
}

\author{
Tiago J. T. Souza ${ }^{1}$, Miriam A. Apel', Sérgio Bordignon ${ }^{2}$, Nelson Ivo Matzenbacher ${ }^{3}$, José $^{\prime}$ \\ Angelo S. Zuanazzi ${ }^{1}$, Amélia T. Henriques ${ }^{1 *}$ \\ 'Programa de Pós-Graduação em Ciências Farmacêuticas, Faculdade de Farmácia, UFRGS, Av. Ipiranga 2752, \\ 90610-000, Porto Alegre, RS, Brasil, \\ ${ }^{2}$ Curso de Ciências Biológicas, Centro Universitário La Salle, Av. Victor Barreto 2288, 92010-000, Canoas, RS, \\ Brasil, \\ ${ }^{3}$ Departamento de Biologia, Faculdade de Biociências, PUCRS, Av. Ipiranga 6681, 90619-900, Porto Alegre, RS, \\ Brasil
}

\begin{abstract}
RESUMO: Os óleos voláteis das folhas e inflorescências de Eupatorium polystachyum DC. (Asteraceae), coletado no Rio Grande do Sul, Brasil, foram caracterizados por CG-EM e sua atividade antioxidante foi avaliada utilizando-se o método de DPPH. Foram obtidos rendimentos de 1,6 e $0,9 \%$ de óleo em relação às folhas e inflorescências frescas, sendo identificados 34 compostos, correspondendo a 99,1 e $99,5 \%$ do óleo volátil, respectivamente. Os óleos apresentaram exclusivamente compostos terpênicos, sendo que as folhas contêm grande quantidade de monoterpenos $(66,4 \%)$ enquanto que as inflorescências apresentaram quantidades equivalentes de mono e sesquiterpenos (51,8 e 47,7\%, respectivamente). As amostras apresentaram-se qualitativamente muito semelhantes sendo constituídas majoritariamente por: $\beta$ pineno (14,7 e 9,8\%), $\beta$-mirceno (15,3 e 10,8\%) e limoneno (22,8 e 20,5\%) entre os monoterpenos e $\beta$-cariofileno (10,4 e 15,4\%), germacreno D (7,2 e 9,4\%) e biciclogermacreno (12,0 e 19,2\%) entre os sesquiterpenos. As diferenças entre as amostras foram basicamente quantitativas. Os óleos apresentaram atividade antioxidante pelo ensaio com DPPH por bioautografia na diluição de 1:10 em éter etílico $(\mathrm{v} / \mathrm{v})$.
\end{abstract}

Unitermos: Eupatorium polystachyum, óleos voláteis, atividade antioxidante, DPPH.

\begin{abstract}
Chemical composition and antioxidant activity of the volatile oil from Eupatorium polystachyum DC". The essential oil obtained from leaves and flowers of Eupatorium polystachyum DC. (Asteraceae) collected in Rio Grande do Sul State, Brazil, was characterized by GC-MS and its antioxidant activity was assessed by DPPH assay. The yield of essential oil, relative to fresh material, was 1.6 and $0.9 \%$ for leaves and flowers, respectively. Both oils are constituted exclusively by terpenic compounds. Leaf oil contains great quantity of monoterpenes (66.4\%); on the other hand, inflorescence oil contains comparable quantities of mono- and sesquiterpenes ( 51.8 and $47.7 \%$, respectively). The same compounds are majority in both oils: $\beta$-pinene (14.7 and 9.8\%), $\beta$-myrcene (15.3 and 10.8\%) and limonene (22.8 and $20.5 \%$ ) among monoterpenes and $\beta$-caryophyllene (10.4 and 15.4\%), germacrene D (7.2 and 9.4\%) and bicyclogermacrene (12.0 and 19.2\%) among sesquiterpenes. The oils had antioxidant activity by DPPH assay in 1:10 dilution in ethyl ether $(\mathrm{v} / \mathrm{v})$.
\end{abstract}

Keywords: Eupatorium polystachyum, essential oils, antioxidant activity, DPPH.

\section{INTRODUÇ̃̃O}

Os óleos voláteis de plantas são conhecidos e utilizados desde a Antigüidade por suas propriedades biológicas, especialmente antibacteriana, antifúngica e antioxidante (Deans; Waterman, 1993). Além disso, as evidências de que alguns compostos antioxidantes sintéticos largamente utilizados na indústria podem promover o desenvolvimento de células tumorais (Botterweck et al., 2000) tem levado a um aumento crescente na procura de similares naturais, dentre estes os óleos voláteis constituídos por compostos terpênicos com importante atividade antioxidante (Candan et al., 2003; Sacchetti et al., 2005). Aliado a isso, o grande interesse das indústrias farmacêutica, alimentícia e cosmética na utilização de novos óleos voláteis e a receptividade dos consumidores para produtos de origem natural transformaram a avaliação sistemática desses produtos vegetais em uma ferramenta muito utilizada na busca de novos compostos com atividade antioxidante. Tais compostos são amplamente estudados por serem capazes de proteger os sistemas 
biológicos, especialmente membranas lipídicas, dos danos produzidos pelo estresse oxidativo, considerado a principal causa do envelhecimento, das doenças degenerativas e do câncer (Cozzi et al., 1997).

Além dessas doenças, os processos inflamatórios também envolvem espécies reativas do oxigênio, originadas pela ativação de leucócitos durante a degranulação. Os óleos voláteis podem atenuar esses processos através da captura dos radicais hidroxila gerados (Graßmann et al., 2000). Sendo assim, um screening de atividade antioxidante também pode fornecer indicações importantes sobre o potencial antiinflamatório de um óleo volátil.

O gênero Eupatorium (Asteraceae) compreende aproximadamente 600 espécies, a maioria delas nativas da América do Sul, das quais mais de 250 são nativas do Brasil e 49 foram descritas no Rio Grande do Sul. A espécie Eupatorium polystachyum DC., seção Subinbricata (DC.) HOFFM., é um arbusto de 1-3 m de altura, ramoso, de flores brancas, cuja floração ocorre no período que vai de janeiro a maio. Essa espécie ocorre no Centro, Sul e Nordeste do Brasil, desde o Piauí até o Rio Grande do Sul, e em países vizinhos, como Peru, Bolívia, Paraguai e Nordeste da Argentina. Baccharis crenulata Spreng. e Raulinoreitzia crenulata (Spreng.) R.M. King \& H. Rob. são considerados seus sinônimos botânicos (Matzenbacher, 1979).

Muitas espécies do gênero Eupatorium já foram avaliadas quanto aos seus componentes químicos voláteis e atividades biológicas, (Albuquerque et al., 2001; Albuquerque et al., 2004; Ding et al., 1994; Habtemariam; MacPherson, 2000; Mongelli et al., 1996; Mongelli et al., 2000; Paolini et al., 2005; Sharma et al., 1998; Souza et al., 2005; Souza et al., 2006; Tori et al., 2001). Os componentes majoritários dos óleos voláteis das espécies já estudadas são terpenos hidrocarbonados como $\alpha$-pineno, $\beta$-pineno, limoneno, sabineno, $\alpha$-felandreno, germacreno $D, \beta$-cariofileno e biciclogermacreno (Paolini et al., 2005; Maia et al., 2002); terpenos oxigenados como espatulenol e globulol (Maia et al., 2002), além de cromenos (Albuquerque et al., 2004), também são descritos para o gênero e são apontados como componentes majoritários em algumas espécies. Igualmente têm sido relatadas variações decorrentes da coleta em diferentes locais para algumas espécies.

Diversos ensaios foram desenvolvidos para screening de atividade antioxidante de extratos vegetais. Os mais utilizados são o ABTS (ácido 2,2'-azinobis-(3etil-benzotiazolino-6-sulfônico)), DPPH (2,2-difenil1-picrilhidrazila), redução do íon férrico (FRAP) e capacidade de absorção de radical oxigênio (ORAC) (Leon; Shui, 2002; Thaipong et al., 2006). Como alguns desses ensaios possuem mecanismos diferentes, sua resposta depende do tipo e da relação oxidante/ antioxidante utilizados. O ensaio de descoloração do radical $\mathrm{DPPH}^{*}$ em que ocorre captura de e radicais formação de DPPH-H é bastante robusto e independe da polaridade do extrato para exercer sua atividade, já tendo sido utilizado no screening de outras espécies de Eupatorium e no ensaio de atividade antioxidante de diversos óleos voláteis de utilização medicinal e alimentícia (Parejo et al., 2003; El-Massry et al., 2002).

Considerando a possibilidade de identificar substâncias com potente atividade biológica, o objetivo deste trabalho foi identificar a composição química do óleo volátil das folhas e das inflorescências de $E$. polystachyum DC., e avaliar o seu potencial antioxidante pelo ensaio de captura de radicais DPPH. Este estudo faz parte de uma investigação sistemática de espécies brasileiras do gênero Eupatorium.

\section{MATERIAL E MÉTODOS}

\section{Material vegetal}

O material vegetal foi coletado durante a floração da espécie E. polystachyum em São Francisco de Paula, região serrana do Rio Grande do Sul, Brasil, em abril de 2005. Uma exsicata foi depositada no Herbário da Universidade Federal do Rio Grande do Sul (ICN) (Bordignon 3095).

\section{Extração do óleo volátil}

Os óleos voláteis foram obtidos das folhas e inflorescências, separadamente, por hidrodestilação durante 4 horas, utilizando um aparelho tipo-Clevenger. $\mathrm{O}$ rendimento do óleo volátil foi calculado em função do volume de óleo obtido e do peso de material vegetal fresco. Para a análise, os óleos voláteis obtidos foram diluídos em éter etílico na razão de 2:100 (v/v).

\section{Análise do óleo volátil}

A análise em CG foi realizada em um cromatógrafo Shimadzu GC17-A equipado com detector de ionização em chama (DIC), usando coluna capilar de sílica fundida DB-5 (25 m x 0,25 mm d.i., 0,25 $\mu \mathrm{M}$ de espessura do filme). Foi utilizado Hélio como gás carreador, a um fluxo de $1,0 \mathrm{~mL} / \mathrm{min}$ e pressão de 80 $\mathrm{kPa}$. A temperatura do forno foi programada de $60^{\circ}$ a $300^{\circ}$ a $3{ }^{\circ} \mathrm{C} / \mathrm{min}$. As temperaturas do injetor e do detector foram de $220{ }^{\circ} \mathrm{C}$ e $250{ }^{\circ} \mathrm{C}$, respectivamente. A razão de fluxo do injetor foi de 1:20. Nitrogênio, ar sintético e hidrogênio foram utilizados como gases auxiliares, na razão de 1:1:10, respectivamente. A composição percentual foi obtida por integração eletrônica pela técnica de normalização utilizando software CR10 (Shimadzu).

A análise por Cromatografia a Gás Espectrometria de Massa (CG-EM) foi realizada em um sistema Shimadzu QP-5000-Quadrupole MS, operando com energia de ionização de $70 \mathrm{eV}$. Foi utilizada coluna

Rev. Bras. Farmacogn. Braz J. Pharmacogn 17(3): Jul./Set. 2007 
capilar de sílica fundida DB-5 (25 m x 0,25 mm d.i., 0,25 $\mu \mathrm{M}$ de espessura do filme); Hélio como gás carreador, fluxo de $1 \mathrm{~mL} / \mathrm{min}$ com split. As temperaturas do injetor e do detector foram de $220^{\circ} \mathrm{C}$ e $250{ }^{\circ} \mathrm{C}$, respectivamente. A temperatura da coluna foi programada de $60{ }^{\circ} \mathrm{C}$ a 300 ${ }^{\circ} \mathrm{C}$ a $3{ }^{\circ} \mathrm{C} / \mathrm{min}$, e os espectros de massa foram obtidos de 30 a $400 \mathrm{~m} / \mathrm{z}$.

A identificação dos componentes dos óleos foi realizada por comparação de seus espectros de massa, bem como de seus índices de retenção (IR), calculados utilizando-se como padrões uma série homóloga de hidrocarbonetos $\mathrm{C} 8$ a $\mathrm{C} 22$, com dados da espectroteca NIST (National Institute of Standards and Technology) ou com dados espectrais da literatura (Adams, 2001, Apel et al., 2005, 2006).

\section{Avaliação da atividade antioxidante}

A atividade antioxidante de cada óleo volátil foi avaliada em cromatoplacas de cromatografia em camada delgada, com gel de sílica, utilizando DPPH (2,2'-difenil-1-picrilhidrazila) como reagente. Foram preparadas três diluições do óleo em éter etílico, nas proporções de 1:10, 1:100 e 1:1000 (v/v). Como substância de referência foi utilizada a quercetina dissolvida em metanol $(1 \mathrm{mg} / \mathrm{mL})$. Foram aplicados $10 \mu \mathrm{L}$ de cada diluição dos óleos e da substância de referência sobre a cromatoplaca. Após evaporação do solvente a placa foi nebulizada com a solução de DPPH. Após 30 min foi realizada a leitura da placa, em que halos brancos ou amarelados no ponto de aplicação, sobre o fundo violeta, são considerados positivos. O ensaio avaliou a capacidade do óleo de prevenir a oxidação do DPPH (Cavin et al., 1998).

\section{RESULTADOS}

Os óleos voláteis foram obtidos por hidrodestilação das folhas e inflorescências de $E$. polystachyum com rendimento de 1,6 e 1,9\% em relação peso de material fresco utilizado, respectivamente. Pela análise em CG-EM foi possível identificar 34 componentes, correspondendo a 99,1 e $99,5 \%$ do óleo volátil. No óleo das folhas foi caracterizada grande quantidade de monoterpenos $(66,4 \%)$ enquanto no óleo das inflorescências observaram-se quantidades equivalentes de mono e sesquiterpenos (51,8 e 47,7\%).

Os mesmos compostos são majoritários em ambas as amostras (folhas e inflorescências, respectivamente): $\beta$-pineno (14,7 e 9,8\%), $\beta$-mirceno $(15,3$ e $10,8 \%)$ e limoneno $(22,8$ e $20,5 \%)$ entre os monoterpenos e $\beta$-cariofileno (10,4 e 15,4\%), germacreno D (7,2 e 9,4\%) e biciclogermacreno (12,0 e $19,2 \%$ ) entre os sesquiterpenos (Tabela 1 ). O ensaio de atividade antioxidante frente ao radical $\mathrm{DPPH}^{\circ} \mathrm{em}$ placas cromatográficas de gel de sílica revelou atividade de captura de radicais livres na diluição de 1:10 (v/v).

\section{DISCUSSÃO}

Óleos voláteis são metabólitos vegetais secundários produzidos pelas plantas por outras necessidades que não a de nutrição, por exemplo, para a atração e repelência de insetos e ação alelopática. A sua produção está integrada à fisiologia de toda a planta, por isso sua composição e quantidade dependem das enzimas específicas que catalisam a produção de compostos voláteis em um órgão, do estágio de desenvolvimento e de estresses abióticos, como a salinidade do solo, a umidade e a temperatura (Sangwan et al., 2001).

O óleo volátil de E. polystachyum é composto exclusivamente por derivados mono- e sesquiterpênicos, não tendo sido identificados compostos voláteis de outras rotas metabólicas, como os derivados fenilpropanóides e compostos alifáticos. Também não foram identificados cromenos, substâncias comuns em algumas espécies de Eupatorium, e sesquiterpenos furânicos, característicos de E. laevigatum (seção Cylindrocephala). A composição química dos óleos analisados não diferiu significativamente da composição já relatada para outras espécies do gênero, apesar de seu rendimento ser maior.

Os seis compostos majoritários que caracterizam o óleo das folhas e das inflorescências foram os mesmos ( $\beta$-pineno, $\beta$-mirceno, limoneno, $\beta$-cariofileno, biciclogermacreno e germacreno $D)$, havendo apenas diferenças quantitativas entre eles. Alguns destes compostos apresentam interessantes atividades biológicas, como o limoneno e o germacreno $\mathrm{D}$ funcionam que funcionam como sinalizadores fundamentais no relacionamento de plantas e insetos (Petrakis et al., 2005). Ensaios farmacológicos demonstraram ação anestésica para o $\beta$-cariofileno (Ghelardini et al., 2001), e analgésica para o -mirceno (Lorenzetti et al., 1991). $\beta$-pineno possui ação antiséptica e antimicrobiana. Além disso, todos os compostos identificados como majoritários no óleo volátil de $E$. polystachyum possuem aplicação como precursores químicos para a semissíntese de compostos voláteis com atividade biológica.

Muitos compostos de origem vegetal possuem potente atividade antioxidante. Recentemente foi comprovada a ação provedora de termotolerância, fotoprotetora e antioxidante de monoterpenos de plantas, relacionadas especialmente à sua capacidade de captar radicais de oxigênio oriundos do processo fotossintético (Peñuelas; Llusia, 2002; Peñuelas; Munné-Bosch, 2005). O ensaio com DPPH ${ }^{\circ}$ demonstrou que na diluição de 1:10 em éter etílico $(\mathrm{v} / \mathrm{v})$ tanto o óleo volátil das folhas coma das inflorescências de $E$. polystachyum é capaz de capturar os radicais existentes, neutralizando-os. Pelo resultado obtido e considerando a similaridade na composição química da cada óleo, observa-se que a diferença quantitativa existente entre os principais constituintes da cada amostra não 
Tabela 1. Composição química do óleo volátil das folhas e inflorescências de Eupatorium polystachyum DC.

\begin{tabular}{|c|c|c|c|c|c|c|c|}
\hline Composto & IR & $\begin{array}{c}\text { Folhas } \\
(\%)\end{array}$ & $\begin{array}{c}\text { Inflor. } \\
(\%)\end{array}$ & Composto & IR & $\begin{array}{c}\text { Folhas } \\
(\%)\end{array}$ & $\begin{array}{c}\text { Inflor } \\
(\%)\end{array}$ \\
\hline santolinatrieno & 901 & 0,3 & 0,7 & $\beta$-elemeno & 1380 & 0,1 & 0,2 \\
\hline$\alpha$-tujeno & 919 & 0,3 & 0,1 & $\beta$-cariofileno & 1406 & 10,4 & 15,4 \\
\hline$\alpha$-pineno & 926 & 5,8 & 3,0 & aromadendreno & 1424 & 0,4 & 0,2 \\
\hline sabineno & 965 & 3,8 & 2,3 & $\alpha$-humuleno & 1438 & 0,6 & 0,8 \\
\hline$\beta$-pineno & 969 & 14,7 & 9,8 & $\gamma$-muuroleno & 1464 & $\operatorname{tr}$ & \\
\hline$\beta$-mirceno & 986 & 15,3 & 10,8 & germacreno D & 1465 & 7,2 & 9,4 \\
\hline$\delta$-2-careno & 994 & 0,4 & 0,9 & biciclogermacreno & 1481 & 12,0 & 19,2 \\
\hline$\alpha$-felandreno & 998 & 0,1 & 0,2 & $\alpha$-muuroleno & 1485 & $\operatorname{tr}$ & 0,3 \\
\hline$\alpha$-terpineno & 1010 & 0,1 & $\operatorname{tr}$ & germacreno A & 1488 & 0,2 & 0,5 \\
\hline$p$-cimeno & 1017 & 0,4 & 0,5 & $\gamma$-cadineno & 1497 & 0,2 & \\
\hline limoneno & 1024 & 22,8 & 20,5 & $\delta$-cadineno & 1508 & 0,9 & 1,0 \\
\hline$(Z)$ - $\beta$-ocimeno & 1031 & 0,3 & 0,5 & espatulenol & 1568 & 0,4 & 0,5 \\
\hline$(E)$ - $\beta$-ocimeno & 1041 & 1,7 & 2,5 & óxido de cariofileno & 1573 & $\operatorname{tr}$ & \\
\hline$\gamma$-terpineno & 1051 & 0,2 & $\operatorname{tr}$ & óxido de humuleno I & 1584 & 0,3 & 0,2 \\
\hline terpinoleno & 1081 & $\operatorname{tr}$ & $\operatorname{tr}$ & & & & \\
\hline terpinen-4-ol & 1168 & 0,2 & $\operatorname{tr}$ & MTH & & 66,2 & 51,8 \\
\hline$\alpha$-terpineol & 1183 & $\operatorname{tr}$ & & MTO & & 0,2 & 0 \\
\hline acetato de nerila & 1354 & $\operatorname{tr}$ & $\operatorname{tr}$ & STH & & 31,0 & 47,0 \\
\hline$\alpha$-copaeno & 1364 & $\operatorname{tr}$ & & STO & & 0,7 & 0,7 \\
\hline$\beta$-bourboneno & 1372 & $\operatorname{tr}$ & & Total & & 98,1 & 99,5 \\
\hline
\end{tabular}

IR = Índice de retenção; Inflor. = Inflorescências; MTH = Monoterpenos hidrocarbonados, MTO = Monoterpenos oxigenados; STH $=$ Sesquiterpenos hidrocarbonados; $\mathrm{STO}=$ Sesquiterpenos oxigenados.

influenciou na resposta da inibição frente ao DPPH. Mesmo as amostras apresentado os mesmos compostos majoritários não é possível inferir com precisão que sejam estes os responsáveis pela ação observada, pois o efeito antioxidante dos óleos voláteis, muitas vezes, decorre do sinergismo existe entre as substâncias (Candan et al., 2003).

\section{CONCLUSÃO}

O óleo volátil das folhas e inflorescências de E. polystachyum DC. foi analisado pela primeira vez. Foram identificados 34 componentes, contabilizando $99,1-99,5 \%$ do total. O óleo apresentou atividade antioxidante pelo ensaio de captura de radicais $\mathrm{DPPH}^{*}$. Estudos posteriores poderão incluir a identificação do(s) composto(s) responsável(is) por essa ação, bem como a avaliação do potencial antiinflamatório desses óleos.

\section{REFERÊNCIAS}

Adams RP 2001. Identification of essential oil components by gas chromatography/quadrupole mass spectroscopy. Allured Publishing Corporation.

Albuquerque MRJR, Souza EB, Mesquita EF, Nunes EP, Cunha AN, Silveira ER 2001. Volatile constituents from leaves of Vernonia chalybaea Mart. and Eupatorium ballotaefolium HBK. J Essent Oil Res 13: 376-377. Albuquerque MRJR, Silveira ER, Uchôa DEA, Lemos TLG, Souza EB, Santiago GMP, Pessoa ODL 2004. Chemical composition and larvicidal activity of the essential oils from Eupatorium betonicaeforme (D.C.) Baker (Asteraceae). J Agric Food Chem 52: 6708-6711.

Apel MA, Sobral M, Zuanazzi JA, Henriques AT 2006. Essential oil compostition of four Plinia species (Myrtaceae). Flav Frag J 21: 565-567.

Apel MA, Sobral M, Sachapoval EES, Henriques AT, Menut C, Bessiere JM 2005. Volatile constituents of Eugenia mattosii Legr (Myrtaceae) J Essent Oil Res 3: 284285.

Botterwerck AAM, Verhagen H, Goldbohm RA, Kleinjans J, Brandt PA 2000. Intake of butylated hydroxyanisole and butylated hydroxytoluene and stomach cancer risk: results from analyses in the Netherlands cohort study. Food Chem Toxicol 38: 599-605.

Candan F, Unlu M, Tepe B, Daferera D, Polissiou M, Sökmen A, Akpulat HA 2003. Antioxidant and antimicrobial activity of the essential oil and methanol extracts of Achillea millefolium subsp. millefolium Afan. (Asteraceae). J Ethnopharmacol 87: 215-220.

Cavin A, Potterat O, Wolfender JL, Hostettman K, Dyatmyko W 1998. Use ofon-flow LC/H-1 NMR for the study of an antioxidant fraction from Orophea enneandra and isolation of a polyacetylene, lignans, and a tocopherol derivative. J Nat Prod 61: 1497-1501. 
Cozzi R, Ricordy R, Aglitti T, Gatta V, Perticone P, De Salvia R 1997. Ascorbic acid and b-carotene as modulators of oxidative damage. Carcinogenesis 18: 223-228.

Deans SG, Waterman PG 1993. Biological Activity of Volatile Oils. In: Hay RKM, Waterman GP (Ed). Volatile oil crops: their biology, biochemistry and production. Londres: John Willey \& Sons.

Ding JK, Yu XJ, Yu W, Ding ZH, Chen ZL, Hayashi N, Komae H 1994. Aromatic components of the essential oils of 4 Chinese medicinal-plants (Asarum petelotii, Elsholtzia souliei, Eupatorium adenophorum, Micromeria biflora) in Yunnan. Z Naturforsch C 49: 703-706.

El-Massry KF, El-Ghorab AH, Farouk A 2002. Antioxidant activity and volatile components of Egyptian Artemisia judaica L. Food Chem 79: 331-336.

Ghelardini C, Galeotti N, Mannelli LC, Mazzanti G, Bartolini A 2001 . Local anaesthetic activity of $\beta$-caryophyllene. Il Farmaco 56: 387-389.

Graßmann J, Hippeli S, Dornisch K, Rohnert U, Beuscher N, Elstner EF 2000. Antioxidant properties of essential oils. Possible explanations for their anti-inflammatory effects. Arzneimittel-Forsch 50: 135-139.

Habtemarian S, MacPherson AM 2000. Cytotoxicity and antibacterial activity of ethanol extract from leaves of an herbal drug, boneset (Eupatorium perfoliatum). Phytother Res 14: 575-577.

Leong LP, Shui G 2002. An investigation of antioxidant capacity of fruits in Singapore markets. Food Chem 76: 69-75.

Lorenzetti BB, Souza GE, Sarti SJ, Santos Filho D, Ferreira SH 1991. Myrcene mimics the peripheral analgesic activity of lemongrass tea. $J$ Ethnopharmacol 34: 43-48.

Maia JGS, Zoghbi MGB, Andrade EHA, Silva MHL, Luz AIR, Silva JDS 2002. Essential oils composition of Eupatorium species growing wild in the Amazon. Biochem Syst Ecol 30: 1071-1077.

Matzenbacher NI 1979. Estudo taxonômico do gênero Eupatorium L. (Compositae) no Rio Grande do Sul Brasil, 310p. Dissertação de Mestrado - Universidade Federal do Rio Grande do Sul.

Mongelli E, Martino V, Coussio J, Ciccia G 1996. Screening of Argentine medicinal plants using the brine shrimp microwell cytotoxicity assay. Pharm Biol 34: 249254.

Mongelli E, Pampuro S, Coussio J, Salomon H, Ciccia G 2000. Cytotoxic and DNA interaction activities of extracts from medicinal plants used in Argentina. $J$ Ethnopharmacol 71: 145-151.

Paolini J, Costa J, Bernardini AF 2005. Analysis of the essential oil from aerial parts of Eupatorium cannabinum subsp. corsicum (L.) by gas chromatography with electron impact and chemical ionization mass spectrometry. J Chromatogr A 1076: 170-178.

Parejo I, Viladomat F, Bastida J, Rosas-Romero A, Saavedra G, Murcia MA, Jiménez MA, Codina C 2003. Investigation of Bolivian plant extracts for their radical scavenging activity and antioxidant activity. Life Sci 73: 1667-1681.

Peñuelas J, Llusià J 2002. Linking photorespiration, monoterpenes and thermotolerance in Quercus. New Phytologist 155: 227-238.
Peñuelas J, Munné-Bosch S 2005. Isoprenoids: an evolutionary pool for photoprotection. Trends Plant Sci 10: 166169.

Petrakis VP, Roussis V, Papadimitriou D, Vagias C, Tsitsimpikou C 2005. The effect of terpenoid extracts from 15 pine species on the feeding behavioural sequence of the late instars of the pine processionary caterpillar Thaumetopoea pityocampa. Behav Process 69: 303322.

Sacchetti G, Maietti S, Muzzoli M, Scaglianti M, Manfredini S, Radice M, Bruni R 2005. Comparative evaluation of 11 essential oils of different origin as functional antioxidants, antiradicals and antimicrobials in foods. Food Chem 91: 621-632.

Sangwan NS, Farooqi AHA, Shabih F, Sangwan RS 2001. Regulation of essential oil production in plant. Plant Growth Regul 34: 3-21.

Sharma OP, Dawra RK, Kurade NP, Sharma PD 1998. A review of the toxicosis and biological properties of the genus Eupatorium. Nat Toxins 6: 1-14.

Souza TJT, Apel MA, Bordignon SAL, Henriques AT 2005. Composição química do óleo volátil das folhas de Symphyopappus reticulatus Baker. V Reunión de la Sociedad Latino-Americana de Fotoquímica. Montevideo, Uruguay.

Souza TJT, Apel MA, Bordignon SAL, Henriques AT 2006. Composição química e atividade antioxidante do óleo volátil de Eupatorium ivifolium L. var hirsutum e Eupatorium inulifolium HBK. XXIX reunião anual da Sociedade Brasileira de Química. Águas de Lindóia, Brasil.

Thaipong K, Boonprakob U, Crosby K, Cisneros-Zevallos L, Byrne DH 2006. Comparison of ABTS, DPPH, FRAP and ORAC assays for estimating antioxidant activity from guava fruit extracts. J Food Comps Anal 19: 669-675.

Tori M, Ohara Y, Nakashima K, Sono M 2001. Thymol derivatives from Eupatorium fortunei. J Nat Prod 64: 1048-1051. 